Cinémas

Revue d'études cinématographiques

Journal of Film Studies

\title{
Présentation. Invention et réinvention du paysage
}

\section{Antonio Costa}

Volume 12, numéro 1, automne 2001

Le Paysage au cinéma

URI : https://id.erudit.org/iderudit/024864ar

DOI : https://doi.org/10.7202/024864ar

Aller au sommaire du numéro

Éditeur(s)

Cinémas

ISSN

1181-6945 (imprimé)

1705-6500 (numérique)

Découvrir la revue

Citer ce document

Costa, A. (2001). Présentation. Invention et réinvention du paysage. Cinémas,

12(1), 7-14. https://doi.org/10.7202/024864ar d'utilisation que vous pouvez consulter en ligne.

https://apropos.erudit.org/fr/usagers/politique-dutilisation/ 


\section{Présentation. Invention et réinvention du paysage}

\section{Antonio Costa}

Invention: c'est probablement le terme le plus fréquemment utilisé par la littérature théorique et la critique pour rappeler que le paysage n'a pas toujours existé; il résulte d'une activité à la fois cognitive et productive, il est le produit d'une attitude par rapport à la nature et d'une intervention sur celle-ci. Depuis l'étude de Kenneth Clark sur le paysage dans l'art (1949) jusqu'aux plus récentes contributions dans les domaines artistique, littéraire et cinématographique, on a cherché à identifier les temps et les modes à travers lesquels, dans différentes cultures et dans différents pays, a pris forme le paysage: un paysage entendu comme objet de connaissance et de contemplation esthétique puis, par la suite, comme objet de consommation. Un paysage entendu aussi comme domaine d'intervention pour l'humain et comme résultat de l'activité humaine'. En tant que "carrefour où se rencontrent des éléments venus de la nature et de la culture, de la géographie et de l'histoire, de l'intérieur et de l'extérieur, de l'individu et de la collectivité, du réel et du symbolique" (Collot, 1997, p. 5), le paysage est un objet qui requiert une approche interdisciplinaire. Mais en tant que point de rencontre d'un modèle culturel et d'une perception, le paysage au cinéma suppose une double réflexion: sur la nature du paysage et sur la nature du cinéma.

Le regard que porte le cinéma sur le paysage renvoie à une série de modèles culturels, artistiques, sociaux qui sont non pas statiques, mais en évolution constante. Le cinéma contribue également à la production de nouveaux modèles de perception liés 
aux propriétés spécifiques du média et à l'évolution de l'institution cinématographique dans les différents contextes politique, social et culturel. Au cinéma, et grâce à lui, le paysage est constamment réinventé. Les rapports entre le cinéma des origines et le paysage, par exemple, ne se réduisent pas uniquement aux vues paysagistes empruntées à la tradition de la peinture, de la photographie et des spectacles d'optique. Il est vrai que ces vues ont eu une importance constitutive dans l'histoire du cinéma: les vues animées reprennent le nom même d'un genre pictural, photographique et spectaculaire (cf. notamment les spectacles optiques comme le panorama, le diorama, etc.), même si la nature propre au moyen de reproduction poussait dans une direction où l'attraction du mouvement était plus importante que la contemplation du paysage (plus urbain que rural, d'ailleurs). À l'attraction du paysage comme objet de contemplation esthétique (dans ses aspects soit pittoresques, soit sublimes), les spectacles d'optique ajoutaient de nouveaux éléments d'attraction (ou se substituaient à ceux qui étaient déjà en place). Il s'agissait d'éléments liés à la forme et aux dimensions de l'image (écran circulaire, dimensions gigantesques), à la particularité du point de vue adopté (ainsi les vues aériennes ${ }^{2}$ ), à l'introduction de mouvements et de transformations (de point de vue, de conditions de lumière), à l'intrusion de facteurs de surprise (choc produit par des événements imprévus ou catastrophiques ayant une forte valeur spectaculaire). Les effets de réalisme intégral poursuivis par la peinture "before photography" (Galassi, 1981) se conjuguaient avec la conquête d'un "regard mobile" (Aumont, 1989) que le cinéma a hérité de la peinture et a développé dans des directions nouvelles et imprévisibles.

Pour définir le tableau d'ensemble de l'étude des aspects iconographiques du paysage, on peut dégager quatre approches principales:

\section{a) Le paysage en tant qu'attraction}

Le paysage en tant que facteur d'attraction spectaculaire précède l'invention et le développement du cinématographe. Le panorama et le diorama, les tentatives de représenter le mouvement et les variations de la lumière dans le paysage sont anté- 
rieurs au cinéma. À travers le cinéma, "la tentative d'importer le paysage dans les villes ", dont Benjamin parlait en se référant aux panoramas, s'est accomplie. Le cinéma a rapidement cessé d'être une attraction spectaculaire indifférenciée et, par le développement des genres, a fini par codifier l'emploi du paysage. En instaurant, par exemple, un système d'oppositions et de différenciations entre l'espace urbain et l'espace extra-urbain, qui rendait possible la "figurativisation» des conflits prévus par les structures de la narration ainsi que l'expression des valeurs culturelles, sociales et esthétiques. Le cinéma a introduit des changements profonds dans la vision et la conception de l'espace, tout en devenant un facteur de modification du paysage urbain (les salles, leur distribution dans la ville, les enseignes lumineuses, les affiches, etc.). Il a donc été un facteur de conditionnement et de "programmation" du regard, bien au-delà de ses effets dans la seule salle de projection. En outre, le rapport entre paysage et cinéma (un rapport toujours très étroit, du cinéma des origines à celui, "apocalyptico-métropolitain ", d'aujourd'hui) peut être mis en relation avec plusieurs phénomènes de spectacularisation de l'espace urbain.

\section{b) Le paysage en tant que production}

Au cinéma (tout comme dans d'autres domaines), les emplois, fonctions et sens du paysage ne définissent pas seulement le rapport d'une culture avec la nature (selon la célèbre définition de Kenneth Clark), mais impliquent souvent une prise de position sur la nature même de la représentation. L'exigence pressante de "faire naturel» au cinéma, que la représentation du paysage contribue le plus souvent à combler, va dans le sens d'une occultation du travail de production (un travail d'ordre à la fois économique, technologique et culturel), avec les résonances idéologiques que cela suppose. Ce qui est intéressant, de ce point de vue, ce n'est pas l'ensemble des changements que la reproduction du paysage a amené dans le champ de la consommation artistique, mais plutôt cette idée du cinéma en tant que lieu de production de la "scène", du "discours» où s'inscrit le paysage reproduit. Des interventions sur le profilmique aux nouvelles technologies numériques, en passant par les techniques d'intervention les plus 
diverses au niveau proprement filmique, l'ensemble des procédés de (re)production est en passe de devenir l'un des moments essentiels d'une stratégie textuelle, d'un processus de production du sens. Il est toujours possible d'établir une relation entre les techniques adoptées et les sens spécifiques que le paysage peut acquérir dans tel ou tel genre, auprès de tel ou tel auteur, ou bien au sein de telle ou telle tendance. Les aspects les plus manifestes de la production du paysage peuvent être repérés dans le cas d'un paysage entièrement reconstruit en studio, en dur ou par ordinateur (de Méliès à la science-fiction), ou encore par des effets spéciaux (par exemple, les transparences chez Hitchcock), ou bien encore par l'emploi de la "théâtralité " et du "pictural" comme formes symboliques d'une conception particulière de la réalité et du cinéma, comme on le trouve chez certains auteurs (Ophuls, Minnelli, Fellini, etc. ${ }^{3}$ ).

\section{c) Le paysage en tant que composante des caractères originaux des cinématographies nationales}

Les caractères originaux des cinématographies nationales dépendent dans une large mesure du type de développement économique, technique et culturel spécifique à l'institution cinématographique; ils dépendent non seulement des traditions littéraires, artistiques, etc., mais aussi, et de façon non négligeable, du "paysage national", au sens le plus général de l'expression, que le cinéma reproduit et englobe plus ou moins intentionnellement dans son système expressif.

Dans l'évolution d'une cinématographie nationale, il est possible de saisir les constantes géo-anthropologiques du paysage et les éventuelles modifications produites par ces macrophénomènes que sont l'industrialisation, la pollution et la dégradation des aires métropolitaines. Il est également possible de définir les stéréotypes qui se sont stratifiés dans la tradition littéraire et picturale: grâce notamment aux nouveaux besoins expressifs ou aux nouvelles possibilités du média, le cinéma peut les reproduire et confirmer, en quelque sorte, leur valeur et leur statut ou, au contraire, les modifier et donner d'eux une image nouvelle.

Ainsi, les caractères "originaux» du paysage américain, ou du moins ceux que l'on considère tels (par exemple, selon la défini- 
tion du paysage de l'Ouest par Howard Mumford Jones [cité par French, 1973, p. 105] : "astonishment, plenitude, vastness, incongruity and melancholy"), coïncident-ils avec les caractères iconographiques d'une grande partie du cinéma, et en tout cas du cinéma plus typiquement américain.

\section{d) Le paysage en tant que "stimmung" et le paysage d'auteur}

Dans le domaine du cinéma dit d'auteur, un sentiment particulier ("stimmung») envers le paysage peut devenir l'un des éléments essentiels de l'univers figuratif ("Un paysage est un état de l'âme" écrivait le jeune Godard, reprenant la fameuse formule d'Henri Amiel [cité par Aumont, 1997, p. 237]). Ce qui est vrai pour un metteur en scène (le premier Bergman, par exemple, ou Kurosawa) peut s'avérer aussi pour tel ou tel genre fortement codifié. Pensons seulement au western où il est possible de reconnaître, justement dans l'interprétation du paysage, et même si celle-ci reste assujettie aux règles du genre, l'image de marque d'un auteur (ainsi, à propos de Monument Valley dans le cinéma de John Ford, Philippe French [1973, p. 104] a-t-il parlé de personal landscape). Un paysage spécifique (prenons par exemple la plaine du Pô pour le cinéma italien) peut se lier à une "stimmung" particulière qui, malgré la diversité des auteurs et des tendances (d'Ossessione de Visconti à Antonioni et à Bertolucci, pour ne mentionner que les plus importants), unifie des productions cinématographiques fort différentes. Il est aussi possible de prendre l'œuvre d'un metteur en scène comme une sorte de texte unique, continu et, par conséquent, de tracer une sorte de carte, de géographie idéale, qui joint et unifie des lieux et des espaces divers dans un même style et une même interprétation.

Les textes recueillis dans ce numéro traitent du paysage dans les différentes acceptions que nous avons définies ici, sous différents points de vue, et montrent les modifications intervenues dans les modèles culturels et perceptifs en relation avec l'évolution des contextes social et culturel. En ce qui concerne le paysage en tant qu'attraction du cinéma des premiers temps, la contribution de Marco Bertozzi passe en revue des éléments de continuité, mais également de différenciation, entre les vues 
animées et la tradition du vedutismo. Larticle de Viva Paci s'ouvre sur une discussion du concept d' "attraction" aussi bien dans le cinéma des origines (à la suite des propositions de Tom Gunning et d'André Gaudreault) que dans le cinéma de l'avantgarde soviétique des années vingt (Eisenstein). Par la suite, l'idée d'attraction est reprise pour interpréter le rôle du paysage dans le cinéma d'Herzog, dont les films sont souvent des véritables investigations du paysage en tant qu'expression d'une puissance terrible, fascinatrice et énigmatique.

Pierre Sorlin examine l'évolution du paysage italien «entre cinéma et histoire" : à partir de son idée du "visible ", l'auteur souligne la nature de "convention partagée " du paysage et montre les façons dont le paysage se transforme dans le passage du cinéma du fascisme à celui de l'après-guerre. L'analyse s'effectue à partir des stéréotypes les plus facilement reconnaissables et identifiables de l'imagerie du paysage italien (la plaine du Pô, la Toscane, le Mezzogiorno).

En s'occupant de Gianni Amelio, un des auteurs qui ont mis en scène les changements les plus significatifs du paysage géoanthropologique italien de ces dernières années (Ladro di bambini, Lamerica), Silvestra Mariniello analyse la reconquête du paysage comme lieu de l'expérience, étranger au Palais, comme dirait Pasolini, et à l' "histoire de complaisance» dont celui-ci parlait (une sorte de dé-monumentalisation du paysage, dirionsnous). Dans la dimension du paysage-chronique en opposition au paysage-Histoire, Mariniello cueille les signes de ce que nous pourrions appeler, en nous appropriant encore une fois d'un concept pasolinien, une nouvelle mutation anthropologique.

Les liens entre paysage et cinématographie nationale sont loin d'être statiques et immuables. Les configurations mêmes du territoire changent en fonction de l'évolution économique et sociale, mais les modèles de perception et d'interprétation de l'espace changent eux aussi. Jean Mottet et Maurizia N ttali étudient précisément ces questions en relation avec, pour le premier, le cinéma français et, pour la deuxième, le cinéma américain. Par le truchement de l'analyse de La Hizine (1995) de Mathieu Kassowitz, vu comme l'une des expressions les plus significatives du Banlieue-film, Mottet montre la relation entre 
non-lieu (ou mieux encore hors-lieu) et non-paysage, non seulement comme résultat de la dégradation affectant les périphéries urbaines, mais également comme une véritable perte d'horizon identifiable. Mottet pose en outre le problème de la relation entre l'habitat et l'omniprésence des images de la télévision et pose, au fond, la question suivante: "Quelle vision "paysagère" peut avoir une collectivité qui n'a devant les yeux que des images venues d'ailleurs?".

Lanalyse de Dances with Wolves (1990) de Kevin Kostner et de Sunchaser (1996) de Michael Cimino permet à Maurizia Natali de montrer, pour sa part, comment les emblèmes paysagers du cinéma sont révisés à partir des nouveaux modèles de l'écologie, du multiculturalisme et du mysticisme new age. L'hypothèse de l'auteure est que cette révision fait réapparaître, comme dans une sorte de "théâtre de la mémoire", les différentes stratifications et les différents conflits esthétiques et idéologiques qui ont trouvé leur accomplissement dans le cinéma (et qui trouvent aujourd'hui leur renouvellement, ou leur "re-médiation»).

Michèle Garneau parcourt les traits spécifiques de la révolution du direct québécois, en montrant comment l'abandon de la voix off de la tradition documentaire a impliqué un nouveau rapport entre image et parole. La fonction de commentaire, de distanciation et de réflexion, traditionnellement confiée à la voix off, "passe du côté du regard". Ceci comporte un passage de la dimension rationnelle (logos) à la dimension sensible (pathos) de l'image-paysage. Le parcours traditionnel, qui va du pays au paysage, s'effectue ainsi à rebours: le paysage, devenu vide et étranger, retrouve son identité, sa vérité en reprenant contact avec le pays. Le paysage perd de son étrangeté lorsqu'en lui s'inscrit la parole des lieux et de ceux qui les habitent (Pierre Perrault) et lorsqu'en lui s'inscrivent les affections (le paysage intérieur, le "paysage sous les paupières" de Lucie Lambert).

Malgré la variété des objets et des approches, les contributions recueillies dans ce numéro de la revue CiNéMAS partagent l'idée que l'invention du paysage est un processus en continuel déroulement: pour le cinéma des origines comme pour le cinéma contemporain, il s'agit, en réalité, d'une ré-invention, entendue cornme reconquête d'une dimension de l'expérience 
qui demande (et produit) continuellement une ré-adaptation du regard et des modèles de représentation.

\author{
Université de Trieste \\ Université de Bologne
}

\title{
NOTES
}

1. Cf. Cauquelin, 1989; Roger, 1997 (en particulier le chapitre " Naissance du paysage en Occident", p. 64-82); Camporesi, 1992. En ce qui concerne le cinéma, cf. Mottet, 1998 et Mottet (dir.), 1999.

2. Cf. Philippe Dubois, "Le regard vertical ou les transformations du paysage", dans Mottet (dir.), 1999, p. 24-44.

3. La confrontation, dans le cinéma d’Éric Rohmer, de la stylisation "théâtrale " du paysage (voir Perceval le Gallois, 1978) et des effets picturaux obtenus à travers les images numériques (voir L'Anglaise et le duc, 2001) serait, en ce sens, d'un grand intérêt.

4. Cf. Sorlin, 1977.

\section{RÉFÉRENCES BIBLIOGRAPHIQUES}

Aumont, 1989 : Jacques Aumont, L'CEil interminable. Cinéma et peinture, Paris, Librairie Séguier, 1989.

Camporesi, 1992: Piero Camporesi, Le belle contrade. Nascita del paesaggio italiano, Milano, Garzanti, 1992.

Cauquelin, 1989: Anne Cauquelin, L'Invention du paysage, Paris, Librairie Plon, 1989.

Clark, 1976: Kenneth Clark, Landscape into Art, London, John Murray, [1949] 1976.

Collot, 1997 : Michel Collot (dir.), Les Enjeux du paysage, Bruxelles, OUSIA, 1997.

Dubois 1999: Philippe Dubois, "Le regard vertical ou les transformations du paysage ", dans Mottet (dir.), 1999.

French, 1973 : Philippe French, Westerns, London, Secker \& Warburg, 1973.

Galassi, 1981 : Peter Galassi, Before Photography. Painting and the Invention of Photography, New York, The Museum of Modern Art, 1981.

Mottet, 1998 : Jean Mottet, L'Invention de la scène américaine. Cinéma et paysage, Paris et Montréal, L'Harmattan, 1998.

Mottet (dir.), 1999 : Jean Mottet (dir.), Les Paysages du cinéma, Seyssel, Champ Vallon, 1999.

Roger, 1997 : Alain Roger, Court traité du paysage, Paris, Gallimard, 1997.

Sorlin, 1977 : Pierre Sorlin, Sociologie du cinema, Paris, Aubier Montaigne, 1977. 